\title{
Detection of Frequency Deviations for Monitoring of Power Systems
}

\author{
Felix Rafael Segundo Sevilla, Jean Dobrowolski, Artjoms Obushevs and Petr Korba
}

\begin{abstract}
In this work an algorithm for identification of power system frequency deviation is presented. The proposed approach can be used to monitor frequency measurements from syncrophasor measurement units (PMU) and to store data only for important events and save storage in the local server. The detection algorithm use a sliding window that rise a flag if the measured frequency deviates from a predefined set point. If the alarm flag is constant over several sliding windows, an event is captured and locally stored for further analysis. To demonstrate the effectiveness of the proposed approach, real PMU measurements from the Swiss power system are used as input.
\end{abstract}

Index Terms-phasor measurement units, frequency problems, stability, system unbalance, monitoring

\section{INTRODUCTION}

International interest on reduction of greenhouse gas emissions to fight against climate change is pushing towards implementation of energy policies where generation of electricity based on renewable energy sources (RES) plays a key role. Decommissioning of conventional generation such as nuclear power plants as in Switzerland or increase of energy efficiency per capita are also some of the main goals of these policies [1]. These adjustments hope to produce a positive environment impact, however what they are certainly doing is introducing new challenges in the real time operation of the transmission systems. The stochastic behavior of generation based on RES and the resulting reduction of inertia cause difficulties to maintain the balance between generation and consumption, which produce fluctuations on the electrical frequency. The standard frequency value or set point in continental Europe (CE) power system is of $50 \mathrm{~Hz}$, while in other parts of the world such as most of the American continent or in Japan, the standard frequency value is $60 \mathrm{~Hz}$. Regardless of its reference value, frequency must be kept as stable as possible for different reasons such as a) keep time accuracy and b) prevent machinery damage [2].

Regarding time accuracy, there still clocks which are run by the frequency of the power system and not by quartz crystal. If the frequency rise, these clocks run faster and vice versa. Large frequency deviations can then cause time accuracy problems like was the case in early 2018 in the CE power system when due to political issues between Serbia and Kosovo, Serbia neglected to meet Kosovo's demand causing a large frequency drop, which consequently caused that several clocks all over CE area slowed down by up to six minutes [3]. Similarly, large electrical machines can suffer damage if frequency changes abruptly. Additionally to the difficulty to maintain the frequency value, it has been observed in power systems with massive integration of RES, lower frequency drops and faster time to reach these values, for these reasons is important to monitor frequency deviation and abrupt frequency changes as fast as possible [4], [5], [6].
Development and deployment of phasor measurement units (PMUs) in power systems, which measure voltages, currents and frequency, is allowing to have more visibility in realtime and is benefiting transmission system operators (TSOs) to better monitor the network [7].

The fast sampling time of PMUs, e.g. 50 samples per second, provide high accuracy and remote access to these information. Nevertheless, these sophisticated devices generate considerable amounts of memory storage in the local server when collecting historical data, e.g. $10 \mathrm{~GB}$ of memory is required for one month of measurements for one PMU signal. The proposed algorithm can be used to detect events where the frequency rises or drops more than a predefined limit. In this form, only days with relevant events, which has been clearly identified in terms of time of the day and date, can be stored and the rest of the measurements can be discarded in order to realise local storage space. It is worth mention that commercial software has algorithms for event selection, however alternative solutions like the one proposed on this brief, can be used in combination with open source software such as phasor data concentrator (PDC), which only receive PMU streams and lack of algorithms for event detection.

\section{DeteCTION OF FREQUENCY DEVIATIONS}

Frequency measurements $f \in \mathbb{R}^{1 \times p}$ from PMU, where $p$ is the total number of measurements, are store in the form of comma separated values or csv files. These files are then exported in Matlab for further analysis. The flow diagram of the proposed approach is depicted on Figure 1.

The algorithm is based on sliding windows of time $t_{i} \in$ $\mathbb{R}^{1 \times n}$ shifted every $n / 4$, where $n$ is the the size of the window. From equation (1) depicted on Figure 1, the deviation $\Delta f_{i} \in \mathbb{R}^{1 \times n}$ of the frequency $f_{i} \in \mathbb{R}^{1 \times n}$ with respect of a fixed set-point value $f_{\text {set }}$ is calculated for $i=1, \ldots, m$, where $m$ is the number of windows in $p$. PMU devices are prone to experience communication problems, which can lead to lack of measured data.

Equation (2) is then used to prevent corrupted data to be classified as events. When PMU is not receiving any data, the $c s v$ file will have empty cells, which Matlab interpret as zero values. In normal conditions, the frequency within an analyzed window $f_{i}$ has frequency values around $f_{\text {set }}$ and thus, the sum of the absolute value of elements in $\Delta f_{i}$ will lie within a certain range defined by $\epsilon$. On the other hand, if $f_{i}$ contains empty measurements, the sum of elements in $\Delta f_{i}$ will be significantly larger than $\epsilon$.

If the criteria defined in equation (2) is true, the data within the analyzed window is considered corrupted and thus, the information of the $i-t h$ window is not further processed. Additionally, we define the alarm variable $\Lambda_{i} \in \mathbb{R}^{1 \times m}$, which is set to zero when frequency values are within an acceptable 


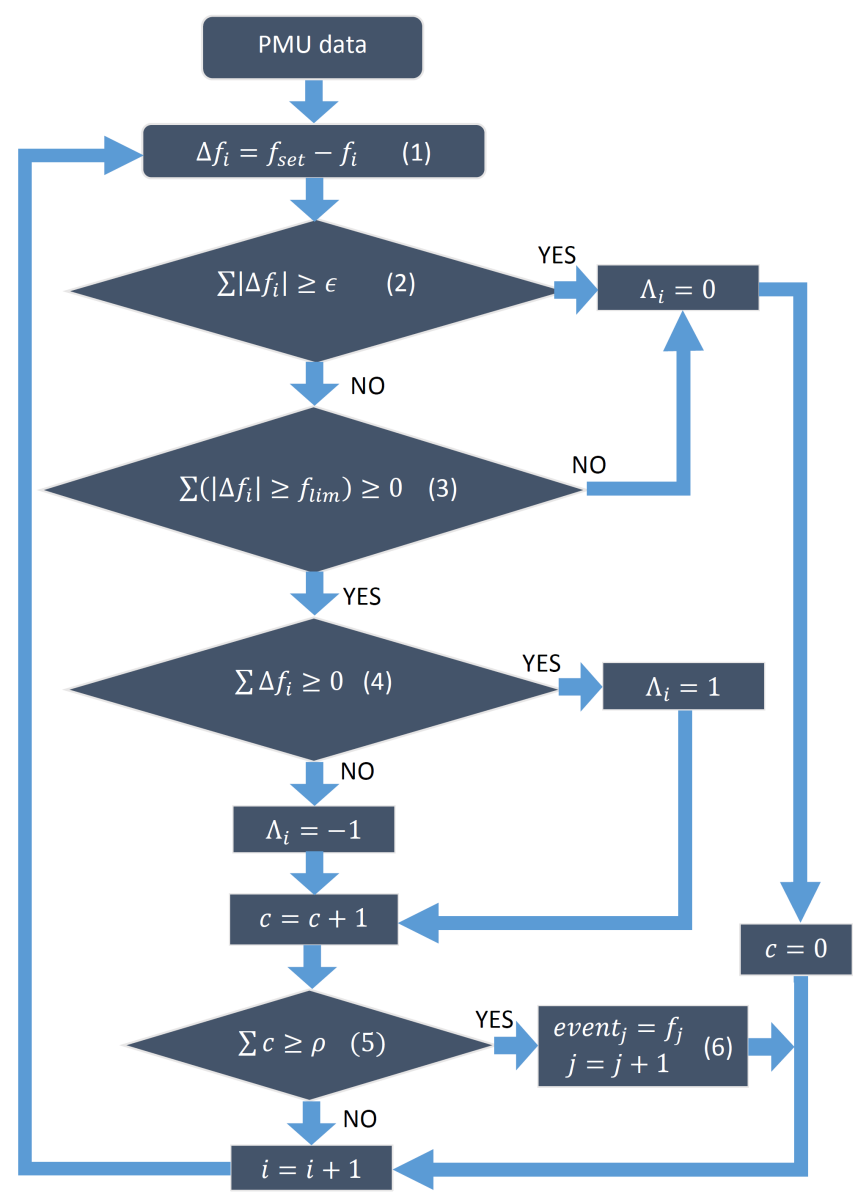

Fig. 1: Flow diagram of the proposed approach.

range or data is corrupted. If equation (2) is false, frequency measurements in $f_{i}$ are reliable and the frequency deviations $\Delta f_{i}$ are compared against a maximum acceptable deviation $f_{\text {lim }}$ as depicted on equation (3). If frequency measurements in $f_{i}$ do not violate the maximum deviation allowed $f_{\text {lim }}$, equation (3) is false and the alarm variable is set to zero $\Lambda_{i}=0$. Conversely, if equation (3) is true, a potential event exist within the analyzed window $f_{i}$ and further steps are required.

Inequality (4) is used to classify the nature of the potential event. If frequency is rising, the alarm variable is set to one $\Lambda_{i}=1$. Similarly, if frequency is dropping, the alarm variable is set to negative unit $\Lambda_{i}=-1$. Regardless the nature of the event, if equation (3) is true, a counter $c$ is trigger. Equation (5) is then used to identify the existence of an actual event. If the counter is bigger or equal to $\rho$, a frequency event has held for a significant period of time. If equation (5) is true, the collection of windows with consecutive alarm flags different to zero contain a frequency event event $_{j}=f_{j} \in \mathbb{R}^{1 \times n * \rho}$, where $j$ is the number of detected events as illustrated in equation (6).

When the alarm variable is set to zero $\Lambda_{i}=0$ the counter is reset $c=0$ preventing false detection and defining the length of detected events. After the alarm variable has been assigned with a corresponding value $[-1,0,1]$, the time window is shifted $i=i+1$ and the detection process start over again.

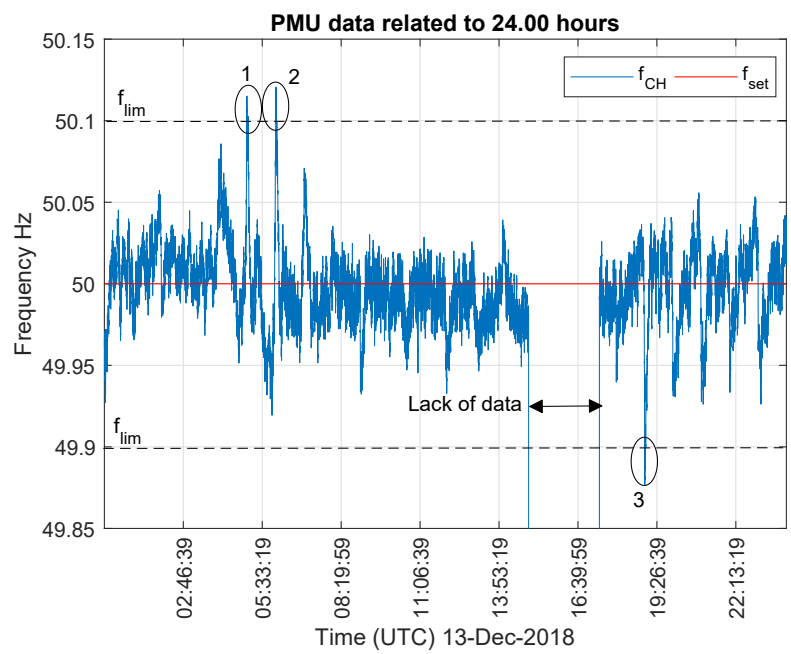

Fig. 2: Real PMU measurement of frequency in Switzerland, recorded over 24 hours the 13th of December 2018 in the ZHAW lab.

\section{Simulation Results}

To demonstrate the effectiveness of the proposed algorithm, real PMU measurements collected from the power systems and smart grid lab of ZHAW, in Switzerland are presented. The installed PMU of national instrument (NI) is connected to the low voltage grid and thus, only frequency measurements are used. The PMU is continuously streaming data (24 hours/ 7 days) to the local PDC, since summer 2017. The storage capacity required to handle the historical data is exponentially growing and thus, the proposed algorithm has been develop to track the most relevant events to be keep in the local storage and delete the irrelevant data.

Figure 2 display the PMU measurement of the frequency in Switzerland during 24 hours, corresponding to the 13th of December 2018. This date was selected because it contains different interesting ingredients such as frequency rises, frequency drops and corrupted data (lack of data). It is worth mentioning that the Swiss power system belongs to the continental Europe (CE) power system and is one of the most reliable power networks in the world, hence, the possibilities to capture or observe a severe event are low. Nevertheless, is possible to monitor minor events related to energy market fluctuations rather than operational issues, which also required special attention to the TSO.

The frequency shown in Figure 2 is given by $f \in \mathbb{R}^{1 \times p}$ for $p=4.32 \times 10^{6}$ corresponding to 24 hours. The size of the time windows was chosen to be of one minute length $n=3000, t_{i} \in \mathbb{R}^{1 \times 3000}$. The window was sliding every $n / 4=750$ samples corresponding to every $15 \mathrm{sec}$. The setpoint was set to $f_{\text {set }}=50 \mathrm{~Hz}$ shown in red on Figure 2. The maximum frequency deviation was set to $f_{\text {lim }}= \pm 110 \mathrm{mHz}$ as depicted on Figure 2 with dotted lines.

The results after applying the algorithm presented on Section II are depicted on Figures 3, 4 and 5, respectively and are highlighted on Figure 2. The algorithm detected 3 events, two frequency rises in the morning and one frequency drop in the evening. Note that the results were not affected despite the large time gap of almost 3 hours, for unknown reasons, where the PMU did not collected any data. 


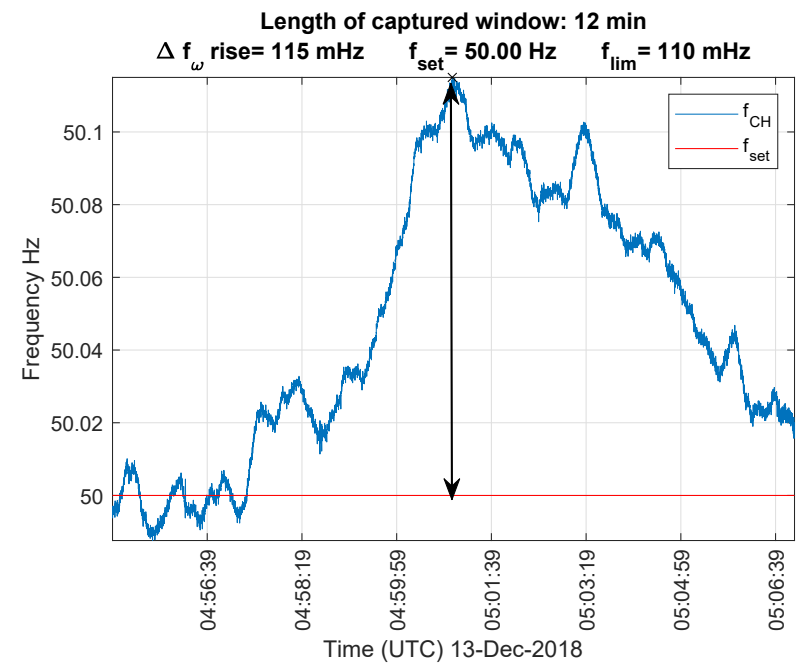

Fig. 3: Frequency rise of more than $110 \mathrm{mHz}$ over $3 \mathrm{~min}$ around $5 \mathrm{am}$.

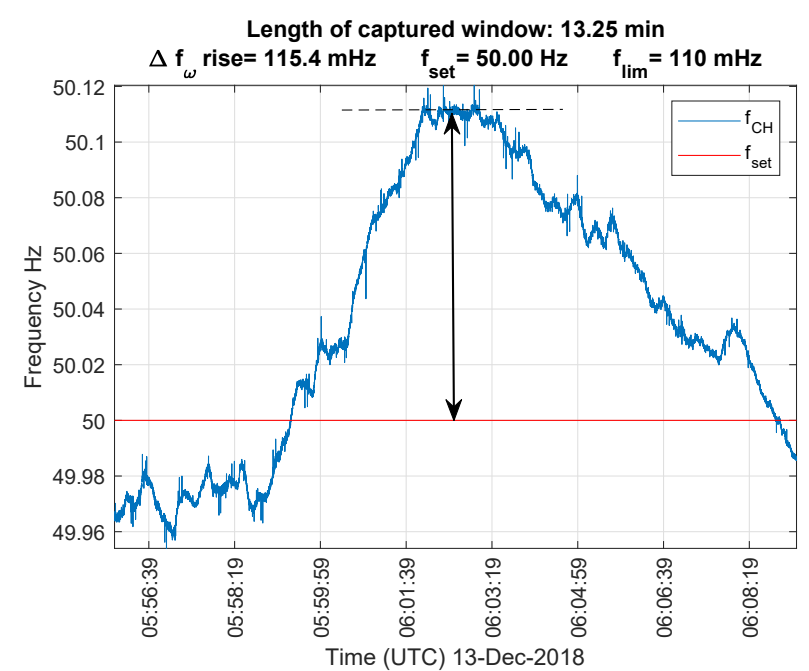

Fig. 4: Frequency rise of more than $110 \mathrm{mHz}$ over $5 \mathrm{~min}$ around $6 \mathrm{am}$.

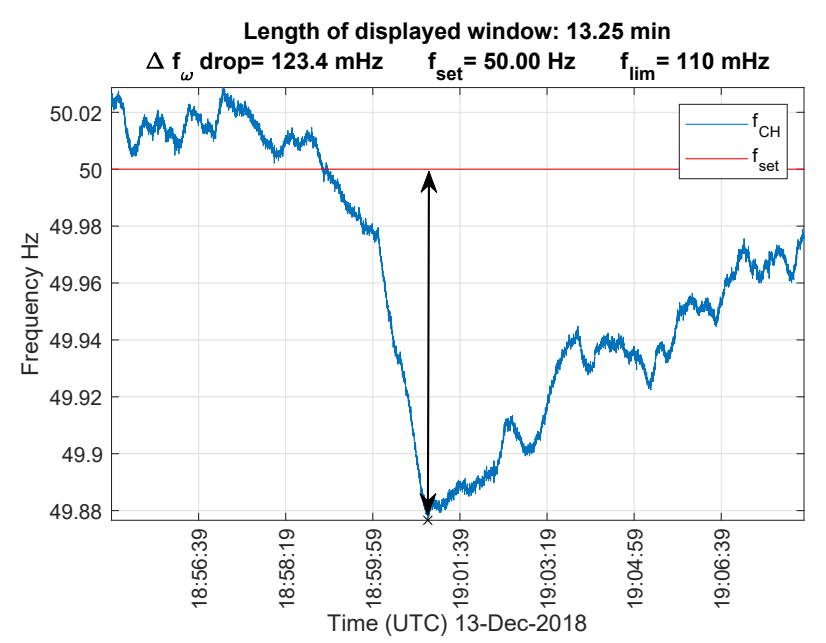

Fig. 5: Frequency drop of more than $120 \mathrm{mHz}$ over $5 \mathrm{~min}$ around $7 \mathrm{pm}$.
During the first event (event $t_{1}$ ), the algorithm captured the violation of the upper frequency limit during 5 consecutive sliding windows. This represented a frequency deviation over a period of $3 \mathrm{~min}$ and the event is displayed on Figure 3. Similarly, two more violations of the frequency limit were observed during 10 consecutive sliding windows for the events displayed on Figures 4 (event 2 ) and 5 (event en $_{3}$, respectively. The last two events represented frequency deviations over a period of $5.5 \mathrm{~min}$. Note that for visualization purposes, Figures 2, 3 and 4 show some extra minutes more before and after the selected event. In this exercise, in order to show the effectiveness of the proposed algorithm, the maximum number of consecutive sliding windows with flag alarm $\rho$ was low. However, the sensitivity of the algorithm can be controlled adjusting to a higher value this parameter, e.g. $\rho=6$. Increasing the value of $\rho$, would result on capturing events of longer duration $(\geqslant 5 \mathrm{~min})$, which in such case event $_{1}$ would be neglected.

\section{Conclusion}

In this paper an algorithm to identify frequency events from PMU measurements has been presented. The proposed has been applied to off-line data stored from measurements, however, the algorithm has been developed to be implemented in real-time in a next stage. Real PMU measurements from the Swiss power system has been used. Simulation results demonstrate that sensitivity of the algorithm can be controlled so the algorithm capture events of short or long duration. Future work include additional frequency analysis such as Rate of Change of Frequency (RoCoF) and application on real-time.

\section{ACKNOWLEDGMENT}

The authors acknowledge the Swiss National Science Foundation (SNSF) under the project number PZENP2 173628 of the program Ambizione Energy Grant (AEG). This research is also part of the activities of SCCER FURIES, which is financially supported by the Swiss Innovation Agency (Innosuisse-SCCER program.

\section{REFERENCES}

[1] Swiss Federal Office of Energy (BFE), "Energy Strategy 2050 (in German)," 2008. [Online]. Available: http://www.bfe.admin.ch/themen/

[2] ENTSO-E, "Entso-e guidance document for national implementation for network codes on grid connection," January 31th, 2018.

[3] ENTSO-E, "Continuing frequency deviation in the continental european power system originating in serbia/kosovo: Political solution urgently needed in addition to technical." March 6th, 2018.

[4] T. Weissbach and E. Welfonder, "High frequency deviations within the european power system: Origins and proposals for improvement," in 2009 IEEE/PES Power Systems Conference and Exposition, March 2009, pp. $1-6$.

[5] A. Dervikadi, Y. Zuo, G. Frigo, and M. Paolone, "Under Frequency Load Shedding based on PMU Estimates of Frequency and ROCOF," in 2018 IEEE PES Innovative Smart Grid Technologies Conference Europe (ISGT-Europe), Oct 2018, pp. 1-6.

[6] Australian Energy Market Operator AEMO, "Black System South Australia 28 September 2016," Technical Report, March 2017.

[7] D. Kim, A. White, and Y. Shin, "Pmu-based event localization technique for wide-area power system," IEEE Transactions on Power Systems, vol. 33, no. 6, pp. 5875-5883, Nov 2018. 